\title{
ORIGINAL
}

\section{DIFERENCIAS EN LOS FACTORES RELACIONADOS CON EL USO DE LA MAMOGRAFÍA EN LAS MUJERES ESPAÑOLAS EN LOS AÑOS 1994 Y $2000(*)$}

\section{Setefilla Luengo Matos, $M^{\text {a }}$ del Mar Polo de Santos}

Agencia de Evaluación de Tecnologías Sanitarias - Instituto de Salud Carlos III.

(*) Este estudio está basado en un proyecto de investigación financiado por el Fondo de Investigación Sanitaria, Instituto de Salud Carlos III. Ministerio de Sanidad y Consumo (FIS 00/218).

\section{RESUMEN}

Fundamento: La mamografía de cribado es el procedimiento más efectivo para la detección precoz del cáncer de mama. El objetivo del presente estudio es conocer las diferencias en los factores relacionados con el uso de mamografía en 1994 y en 2000 en las mujeres españolas de 40-70 años, y en aquéllas con al menos una mamografía en los dos años previos.

Método: Comparación de los resultados de dos encuestas realizadas en 1994 y en 2000 en sendas muestras de mujeres representativas de las mujeres españolas de 40-70 años (3.218 en 1994 y 2.409 en 2000). Se analizaron variables sociodemográficas, socio-sanitarias y de actitudes e información de la mujer.

Resultados: De 1994 a 2000 se encuentran diferencias estadísticamente significativas en las actitudes, destacando la intención de hacerse una mamografía en el futuro (96\% en 2000 vs $79 \%$ en 1994) $(\mathrm{p}<0,0001)$. El porcentaje de realización de la prueba en el grupo de 51-65 años es de 59\% en 2000 vs 50\% en 1994

Conclusiones: El perfil de las mujeres españolas ha cambiado de 1994 a 2000, aunque las mujeres que se habían realizado una mamografía presentan características sociodemográficas y socio-sanitarias similares. No obstante, en el año 2000 la mamografía se realiza en mayor medida en el grupo de 51-65 años de edad y las mujeres muestran actitudes más positivas ante la mamografía.

Palabras clave: Cáncer de mama. Cribado. Mamografía. Conocimientos. Actitudes y Práctica en Salud.

\section{ABSTRACT
Differences in the Factors Related to the Use of Mammograms among Spanish Women in 1994 and 2000

Background: The screening mammography is the most highly effective procedure for early breast cancer detection. This study is aimed at ascertaining the differences in the factors related to Spanish women 40-70 years of age having had mammograms in 1994 and 2000 and among those having had at least one mammogram within the two years prior.

Method: Comparison of the results of two surveys conducted in 1994 and 2000, each on samples of women representative of Spanish women 40-70 years of age (3,218 in 1994 and 2,409 in 2000). An analysis was made of sociodemographic, sociosanitary and the women's attitude and awareness-related variables.

Results: Statistically significant differences were found between 1994 and 2000, the intention of having a mammogram in the future (96\% in 2000 vs. $79 \%$ in 1994) (p<0.0001). A total of $59 \%$ of the $51-$ 65 age group had a mammogram in 2000 , as compared to the $50 \%$ who had one in 1994

Conclusions: The profile of Spanish women has undergone a change from 1994 to 2000 , although those women who had had a mammogram were of similar sociodemographic and sociosanitary characteristics. However, the screening is being done to a greater extent on the age 51-65 age group in 2000, and the women are showing more positive attitudes toward having a mammogram.

Key words: Breast cancer. Mass screening. Mammography. Health knowledge, attitudes, practice.

Correspondencia:

Setefilla Luengo Matos.

Agencia de Evaluación de Tecnologías Sanitarias.

Instituto de Salud Carlos III.

C/ Sinesio Delgado, 4 - Pabellón 4.

28029 Madrid. España.

Correo electrónico: sluengo@isciii.es 


\section{INTRODUCCIÓN}

En la mayoría de los países desarrollados el cáncer de mama constituye la primera causa de muerte por cáncer en la mujer. En España, aunque la incidencia y mortalidad son menores que en otros países europeos, la incidencia ajustada por edad es de $66,81^{1}$ por 100.000 mujeres. Con relación a la mortalidad, en el año 2002 se produjeron 5.751 muertes por cáncer de mama en España, lo que supone una tasa estandarizada ajustada a la población europea de 20,64 por 100.000 mujeres ${ }^{2}$.

La mamografía de cribado se considera el procedimiento más efectivo para la detección precoz del cáncer de mama y la disminución de la mortalidad por la enfermedad. La evidencia científica disponible muestra que la mamografía de cribado en mujeres de 50 a 64 años puede reducir al menos un $20 \%$ la mortalidad por cáncer de mama ${ }^{3,4}$, si bien la efectividad de la prueba depende en gran medida de la edad de la mujer. La mamografía puede beneficiar a las mujeres de 40 a 50 años de edad, aunque fundamentalmente está indicada en las mayores de 50 años de edad $^{5}$. Aparte de los beneficios derivados de la realización de la mamografía el procedimiento implica también ciertos riesgos, siendo los más importantes los relacionados con los diagnósticos falsos positivos. Por ello, se considera fundamental que la mamografía de cribado garantice la máxima calidad para minimizar los efectos adversos de la aplicación masiva de la prueba ${ }^{6}$.

La Unión Europea recomienda la realización de una mamografía cada dos años a las mujeres de 50 a 69 años de edad de la población general ${ }^{7}$. En España se recomienda igualmente la realización periódica de la prueba y se han implantado en los últimos años programas de detección precoz de cáncer de mama en todas las Comunidades Autónomas (CCAA) ${ }^{8}$. Los programas invitan a las mujeres de 50 a 64 años a realizarse cada dos años una mamografía, aunque algu- nos incluyen a mujeres a partir de los 45 años de edad. Recientemente algunos programas están ampliando su cobertura, incluyendo a mujeres hasta los 69 años de edad ${ }^{9}$. Su objetivo común es reducir la mortalidad por cáncer de mama, para lo que precisan conseguir una alta tasa de participación de las mujeres de la población diana ${ }^{8,10}$.

La existencia de programas de detección precoz de cáncer de mama constituye un factor fundamental para que las mujeres se realicen mamografías. En 1994 en España sólo ocho CCAA contaban con programas de detección precoz de cáncer de mama y el $28 \%$ de las mujeres españolas de 40 a 70 años se había realizado al menos una mamografía en los últimos dos años ${ }^{11}$. En el año 2000, con programas de cribado de cáncer de mama en todas las CCAA, la realización de la prueba alcanza al 59\% de las mujeres españolas de 40 a 70 años $^{8}$. Sin embargo, la existencia de programas no garantiza la realización de la prueba ${ }^{12}$. El uso de la mamografía está influido por factores diversos como características sociodemográficas (edad, clase social, situación laboral, nivel de estudios) socio-sanitarias (recomendación del médico, visitas al ginecólogo, cobertura sanitaria) y las actitudes y el nivel de información de la mujer sobre el cáncer de mama y la mamografía ${ }^{13-19}$.

El objetivo del presente estudio es conocer las posibles diferencias en los factores relacionados con el uso de la mamografía en el año 1994 y en el año 2000 en las mujeres españolas de 40 a 70 años, y en aquéllas que se habían realizado al menos una mamografía en los dos años previos.

\section{MATERIAL Y MÉTODOS}

\section{Población de estudio}

Se parte de dos estudios transversales mediante encuesta con base poblacional en una muestra representativa de mujeres de 40 
a 70 años residentes en España, realizados en $1994^{11}$ y en el año $2000^{8}$. En ambos estudios el objetivo fue conocer la proporción de mujeres que se había realizado una mamografía y los factores relacionados con el uso de la prueba. En los dos casos la selección de la muestra se realizó a través de un muestreo por conglomerados en tres etapas, con estratificación previa de las unidades de primera etapa, siendo la unidad muestral de primera etapa la sección censal. Los criterios de estratificación fueron dos: por Comunidad Autónoma en 17 estratos, y por tamaño de municipio de residencia definido por su número de habitantes. Las unidades de segunda etapa fueron los hogares y las de tercera etapa las mujeres de 40 a 70 años residentes en dichos hogares.

Las secciones censales se seleccionaron por muestreo aleatorio simple. Los hogares se seleccionaron mediante rutas aleatorias, realizándose una entrevista por hogar. En el supuesto de que existiera más de una mujer de 40 a 70 años la selección en cada hogar se realizó asignando probabilidad igual a cada una de ellas.

Como se desconocía la proporción de mujeres que se hacían mamografías en España en esos años, para determinar el tamaño de la muestra en los dos estudios se asumió la situación más desfavorable (50\% de realización de mamografía). Se consideró un nivel de confianza del 95\%, y un error de muestreo de $\pm 2 \%$. El tamaño y la distribución muestral de las dos encuestas son diferentes. La tasa de no-respuesta fue del 20,1\% en la encuesta del año 2000 con un tamaño final de la muestra de 2.409 entrevistas, y la tasa de no-respuesta en 1994 fue del 26,5\% con un tamaño final de la muestra de 3.218 mujeres entrevistadas. En el año 2000 la asignación muestral por estrato de comunidad autónoma y tamaño del municipio fue proporcional al número de mujeres de 40 a 70 años residentes en cada estrato, mientras que en el año 1994 se utilizó una asignación no proporcional, partiendo de un mínimo de
100 mujeres en cada Comunidad Autónoma. Las diferencias en el diseño muestral hacen necesario que en el presente estudio se lleve a cabo un ajuste de los datos de 1994 por edad y tamaño de municipio de residencia a la estructura poblacional existente en el estudio del año 2000.

\section{Recogida de información y variables de estudio}

La recogida de información se realizó de marzo a mayo de 1994 en el primer estudio y en octubre de 2000 en el segundo, mediante entrevista personal en el hogar a partir de un cuestionario estructurado. Los cuestionarios utilizados en 1994 y 2000 no son exactamente iguales, por lo que para el presente trabajo se han seleccionado únicamente aquellas variables recogidas de forma similar en ambos estudios.

La variable «realización de la mamografía» se recogió en ambas encuestas preguntando a la mujer si se había realizado al menos una prueba en los dos años previos al momento de la entrevista, independientemente del motivo para su realización: programa de detección precoz de cáncer de mama, indicación del médico o decisión propia. Las variables seleccionadas potencialmente relacionadas con la realización de mamografía son las siguientes:

a) Variables sociodemográficas: edad, estado civil, situación laboral y tamaño del municipio de residencia.

b) Variables socio-sanitarias: cobertura sanitaria (pública, privada/mixta), visitas al ginecólogo en los dos últimos años, visitas al médico general en los dos últimos años, y recomendación de la prueba por parte del médico.

c) Actitudes de la mujer e información sobre el cáncer de mama y la mamografía: intención futura de realización 
de mamografía, percepción de la necesidad de la realización de mamografía aún sintiéndose bien, percepción de posibilidades de curación del cáncer de mama con detección precoz, no dejar de realizarse una mamografía por miedo al diagnóstico de cáncer, y percepción de mayor riesgo de cáncer de mama respecto a otras mujeres de su edad.

d) Otros: historia de enfermedad de mama (en el momento del estudio o anteriormente), familiar directo con cáncer de mama, y otro familiar o amiga con cáncer de mama.

\section{Análisis estadístico}

Se realizó una distribución de frecuencias de las características de las mujeres españolas de 40 a 70 años en el año 1994 y en el año 2000. Se llevó a cabo un estudio comparativo de dichos factores en el año 1994 y en el año 2000, utilizando el test Chi cuadrado $\left(\chi^{2}\right)$ de asociación de variables cualitativas. Igualmente, se realizó un estudio comparativo de las características de la población de mujeres de 40 a 70 años que se habían realizado una mamografía (al menos una prueba en los dos años previos al momento de la entrevista) en 1994 y en el año 2000 utilizando también el test $\chi^{2}$. Se consideraron estadísticamente significativos los valores de $\mathrm{p}<0,05$. El paquete estadístico utilizado para el análisis de datos es el SPSS versión 10.0.

\section{RESULTADOS}

La tabla 1 muestra los factores relacionados con el uso de la mamografía en la población de mujeres españolas de 40 a 70 años de edad en 1994 y en el año 2000. Respecto a las características sociodemográficas destaca la diferencia en el porcentaje de mujeres divorciadas/separadas, que es del $4 \%$ en el año 2000 versus $2 \%$ en 1994 ( $\mathrm{p}=0,008)$, y el porcentaje de mujeres trabajadoras por cuenta ajena, $17 \%$ en 2000 versus $11 \%$ en 1994 ( $<<0,0001)$. Con relación a las características sociosanitarias, el porcentaje de mujeres con cobertura sanitaria privada y mixta es del $11 \%$ en 2000 versus $9 \%$ en 1994 $(\mathrm{p}=0,043)$; el porcentaje de mujeres que habían visitado al ginecólogo y al médico general en los dos últimos años era del 59\% en 2000 versus 44\% en 1994, y $90 \%$ en 2000 versus $84 \%$ en 1994, respectivamente $(\mathrm{p}<0,0001)$; y el porcentaje de mujeres a las que el médico había recomendado una mamografía era del 53\% en 2000 frente al $25 \%$ en 1994 ( $\mathrm{p}<0,0001)$. En cuanto a los factores relacionados con las actitudes de la mujer e información sobre el cáncer de mama y la mamografía se encontraron diferencias significativas en todas las variables estudiadas ( $p<0,0001)$. El $81 \%$ de las mujeres en el año 2000 manifiesta tener intención de realizarse una mamografía en el futuro versus $51 \%$ en 1994 , el $85 \%$ en el año 2000 considera que la mamografía es una prueba necesaria aún sintiéndose bien frente al $78 \%$ en 1994, el 92\% en 2000 piensa que el cáncer de mama tiene más posibilidades de curación con detección precoz frente al $89 \%$ en 1994, y el 94\% en 2000 no dejaría de ir a hacerse una mamografía por miedo al diagnostico de cáncer frente al 89\% en 1994. Igualmente, en el año 2000 el 10\% de las mujeres consideraba que tenía mayor riesgo de padecer cáncer de mama respecto a las mujeres de su edad frente al 7\% en 1994. En relación con «otros factores» el porcentaje de mujeres con familiares directos con cáncer de mama era del $16 \%$ en 2000 y del $12 \%$ en 1994 ( $\mathrm{p}<0,0001)$, y el porcentaje de mujeres con otros familiares o amigos con la enfermedad era del $34 \%$ en 2000 frente al 39\% en 1994 ( $\mathrm{p}<0,0001)$. No se encontraron diferencias estadísticamente significativas en el porcentaje de mujeres con historia personal de enfermedad de mama. Del total de factores estudiados, de 1994 a 2000 destaca el incremento en la intención de la mujer de hacerse una mamografía en el futuro (30\%), la reco- 
Tabla 1

Factores relacionados con el uso de la mamografía en las mujeres españolas de 40 a 70 años, en el año 1994 y año 2000

\begin{tabular}{|c|c|c|c|c|}
\hline & $\begin{array}{c}\text { Año } 2000 \\
\mathrm{n}=2.409 \\
(\%)\end{array}$ & $\begin{array}{c}\text { Año } 1994 \\
\mathrm{n}=3.218 \\
(\%)\end{array}$ & $\chi^{2}$ & $\mathbf{P}$ \\
\hline \multicolumn{5}{|l|}{ Variables Sociodemográficas } \\
\hline Estado civil & & & 11,83 & $=0,008$ \\
\hline soltera & 4,2 & 5,1 & & \\
\hline casada & 80,4 & 80,0 & & \\
\hline divorciada / separada & 3,6 & 2,1 & & \\
\hline viuda & 11,7 & 12,7 & & \\
\hline Situación laboral & & & 41,1 & $<0,0001$ \\
\hline cuenta propia & 5,4 & 6,5 & & \\
\hline cuenta ajena & 17,0 & 10,8 & & \\
\hline no trabaja & 77,6 & 82,7 & & \\
\hline \multicolumn{5}{|l|}{ Variables Socio-sanitarias } \\
\hline Cobertura sanitaria & & & 6,3 & $=0,043$ \\
\hline Pública & 89,5 & 91,2 & & \\
\hline Mixta/Privada & 10,5 & 8,8 & & \\
\hline Visita ginecólogo dos últimos años & 59,2 & 44,1 & 110,60 & $<0,0001$ \\
\hline Visita médico general dos últimos años & 89,8 & 84,0 & 35,37 & $<0,0001$ \\
\hline Recomendación del médico & 52,8 & 25,1 & 394,93 & $<0,0001$ \\
\hline \multicolumn{5}{|c|}{ Actitudes de la mujer e información sobre el cáncer de mama y la mamografia } \\
\hline Intención de hacerse mamografía en el futuro & 80,8 & 50,5 & 490,0 & $<0,0001$ \\
\hline Necesidad de mamografía aún sintiéndose bien & 84,5 & 78,0 & 34,63 & $<0,0001$ \\
\hline Posibilidad de curación con detección precoz & 92,0 & 88,9 & 12,71 & $=0,0001$ \\
\hline No dejaría de ir por miedo al diagnóstico de cáncer & 93,8 & 88,6 & 41,15 & $<0,0001$ \\
\hline $\begin{array}{l}\text { Percepción de mayor riesgo de cáncer de mama respecto a otras } \\
\text { mujeres de su edad }\end{array}$ & 10,4 & 7,1 & 15,9 & $<0,0001$ \\
\hline \multicolumn{5}{|l|}{ Otras variables } \\
\hline Historia de enfermedad de mama & 11,3 & 10,5 & \multicolumn{2}{|c|}{ n.s. } \\
\hline Familiar directo con cáncer de mama & 15,5 & 11,8 & 13,94 & $<0,0001$ \\
\hline Otro familiar o amiga con cáncer de mama & 33,7 & 39,2 & 16,37 & $<0,0001$ \\
\hline
\end{tabular}

mendación del médico (28\%) y la visita al ginecólogo en los dos últimos años (15\%).

Las características de la población de mujeres que se habían realizado una mamografía (al menos una prueba en los dos años previos) en 1994 y en el año 2000 aparecen en la tabla 2. De los factores sociodemográficos estudiados únicamente se encontraron diferencias estadísticamente significativas en la variable edad. La mayor diferencia, aunque en sentido opuesto, corresponde a los grupos de 56 a 60 años y de 46 a 50 años de edad. En cuanto a los factores socio-sani- tarios el porcentaje de mujeres a las que el médico había recomendado una mamografía era del $68 \%$ en 2000 y del $58 \%$ en 1994 ( $\mathrm{p}<0,0001)$, y el porcentaje de mujeres con visitas al médico general era del $92 \%$ en el año 2000 y del 88\% en 1994 ( $\mathrm{p}=0,021)$. No se encontraron diferencias estadísticamente significativas en las variables de cobertura sanitaria, y visita al ginecólogo en los últimos dos años. En relación con los factores de actitudes de la mujer e información sobre el cáncer de mama y la mamografía, el $96 \%$ de las mujeres en el año 2000 manifestaba tener intención de realizarse una mamogra- 
Tabla 2

Factores relacionados con el uso de la mamografía en las mujeres españolas de 40 a 70 años que se habían realizado una mamografía en los dos años previos, año 1994 y año 2000

\begin{tabular}{|c|c|c|c|c|}
\hline & $\begin{array}{c}\text { Año } 2000 \\
(\mathrm{n}=1.427) \\
(\%)\end{array}$ & $\begin{array}{c}\text { Año } 1994 \\
(\mathrm{n}=900) \\
(\%)\end{array}$ & $\chi^{2}$ & $\mathbf{P}$ \\
\hline \multicolumn{5}{|l|}{ Variables Sociodemográficas } \\
\hline Edad & & & 36,27 & $<0,0001$ \\
\hline $40-45$ & 13,5 & 17,2 & & \\
\hline $46-50$ & 16,7 & 24,7 & & \\
\hline $51-55$ & 22,3 & 23,2 & & \\
\hline $56-60$ & 21,2 & 15,1 & & \\
\hline $61-65$ & 15,6 & 11,2 & & \\
\hline$>65$ & 10,7 & 8,6 & & \\
\hline Estado civil & & & & n.s. \\
\hline soltera & 3,0 & 4,4 & & \\
\hline casada & 83,6 & 84,6 & & \\
\hline divorciada / separada & 3,4 & 2,7 & & \\
\hline viuda & 10,0 & 8,3 & & \\
\hline Situación laboral & & & & n.s. \\
\hline cuenta propia & 5,3 & 6,0 & & \\
\hline cuenta ajena & 18,4 & 15,1 & & \\
\hline no trabaja & 76,3 & 78,9 & & \\
\hline Tamaño municipio & & & & n.s. \\
\hline$<2000$ & 7,6 & 8,6 & & \\
\hline $2001-10000$ & 14,9 & 16,3 & & \\
\hline $10001-50000$ & 24,5 & 20,4 & & \\
\hline $50001-100000$ & 9,1 & 7,6 & & \\
\hline$>100000$ & 43,9 & 47,1 & & \\
\hline \multicolumn{5}{|l|}{ Variables Socio-sanitarias } \\
\hline Cobertura sanitaria & & & & n.s. \\
\hline Pública & 86,4 & 86,3 & & \\
\hline Mixta / Privada & 13,6 & 13,7 & & \\
\hline Visita ginecólogo dos últimos años & 79,3 & 82,4 & & n.s. \\
\hline Visita médico general dos últimos años & 91,5 & 88,3 & 5,34 & $=0,021$ \\
\hline Recomendación del médico & 68,1 & 57,9 & 20,78 & $<0,0001$ \\
\hline \multicolumn{5}{|c|}{ Actitudes de la mujer e información sobre el cáncer de mama y la mamografía } \\
\hline Intención de hacerse mamografía en el futuro & 95,7 & 79,3 & 142,92 & $<0,0001$ \\
\hline Necesidad de mamografía aún sintiéndose bien & 95,4 & 89,6 & 24,96 & $<0,0001$ \\
\hline Posibilidad de curación con detección precoz & 94,2 & 92,7 & & n.s. \\
\hline No dejaría de ir por miedo al diagnóstico de cáncer & 98,2 & 95,2 & 16,01 & $<0,0001$ \\
\hline $\begin{array}{l}\text { Percepción de mayor riesgo de cáncer de mama } \\
\text { respecto a otras mujeres de su edad }\end{array}$ & 13,3 & 7,4 & 21,92 & $<0,0001$ \\
\hline \multicolumn{5}{|l|}{ Otras variables } \\
\hline Historia de enfermedad de mama & 15,6 & 22,1 & 13,15 & $<0,0001$ \\
\hline Familiar directo con cáncer de mama & 17,3 & 13,3 & 5,27 & $<0,022$ \\
\hline Otro familiar o amiga con cáncer de mama & 37,5 & 46,3 & 14,52 & $<0,0001$ \\
\hline
\end{tabular}

fía en el futuro versus $79 \%$ en 1994 ( $p<0,0001$ ), el $95 \%$ en 2000 consideraba que la mamografía es una prueba necesaria aún sintiéndose bien versus $90 \%$ en 1994 
Tabla 3

Variables de actitudes de la mujer e información sobre el cáncer de mama y la mamografía por grupos de edad en las mujeres que se habían realizado una mamografía en los dos años previos, en el año 2000

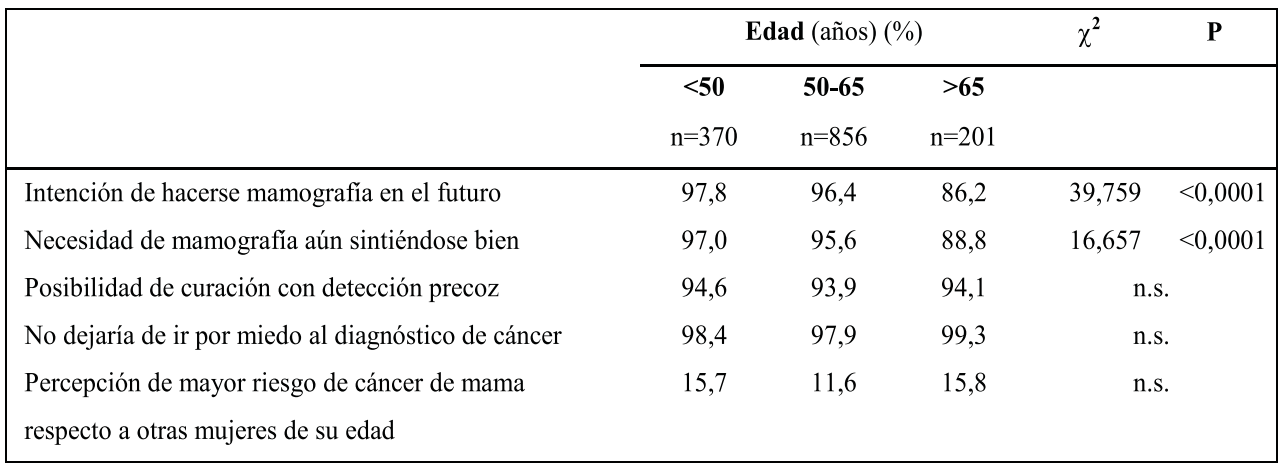

( $\mathrm{p}<0,0001)$, y el $98 \%$ en 2000 no dejaría de ir a hacerse una mamografía por miedo al diagnostico de cáncer frente al 95\% en 1994 $(\mathrm{p}<0,0001)$. No se encontraron diferencias significativas en la percepción de la mujer sobre las posibilidades de curación del cáncer de mama con detección precoz. En relación con Otras variables estudiadas, el porcentaje de mujeres con historia personal de enfermedad de mama es del $16 \%$ en $200022 \%$ en $1994(\mathrm{p}<0,0001)$, el porcentaje de mujeres con familiares directos con cáncer de mama es del 17\% en $200013 \%$ en 1994 ( $\mathrm{p}=0,022)$ y el de mujeres con otros familiares y amigas con cáncer de mama es del $38 \%$ en 2000 46\% en 1994 ( $p<0,0001)$. Del total de factores estudiados, los que presentan mayor incremento de 1994 a 2000 son la intención de hacerse una mamografía en el futuro (17\%), la recomendación de la prueba por parte del médico (10\%) y la edad (6\% en el grupo de 56 a 60 años).

La tabla 3 muestra las variables de actitudes de la mujer e información sobre el cáncer de mama y la mamografía por grupos de edad, en las mujeres que se habían realizado al menos una mamografía en los últimos dos años, en el año 2000. El porcentaje de mujeres con intención de hacerse una mamografía en el futuro es del $86 \%$ en las mujeres mayores de 65 años frente al $98 \%$ en las mujeres menores de 50 años y al $96 \%$ en el grupo de 50 a 65 años (P<0,0001). El 89\% de las mujeres mayores de 65 años considera que la mamografía es necesaria aún sintiéndose bien frente al $97 \%$ en las menores de 50 años y al $96 \%$ en el grupo de 50 a 65 años $(\mathrm{p}<0,0001)$. No se encontraron diferencias estadísticamente significativas en el resto de las variables analizadas.

\section{DISCUSIÓN}

De los resultados de este estudio se desprende que el perfil de la población de mujeres de 40 a 70 años ha cambiado en nuestro país de 1994 al año 2000. Al igual que se recoge en publicaciones oficiales, en nuestro estudio encontramos que determinadas características de las mujeres españolas han variado en los últimos años. Existe una menor tasa de paro, un mayor número de mujeres divorciadas y $\operatorname{separadas}^{20}$, y una mayor proporción de mujeres con cobertura sanitaria privada o mixta, posiblemente en relación con el mayor índice de riqueza en España en el año 2000 en comparación con $1994^{21}$.

De las características sociosanitarias estudiadas llama la atención el aumento signifi- 
cativo del número de mujeres que refiere haber visitado al ginecólogo en los últimos dos años, y el llamativo aumento de la recomendación de la mamografía por parte del médico en el año 2000 en comparación con 1994. En este último año, sólo la cuarta parte de las mujeres españolas manifestaba que su médico les hubiera recomendado alguna vez hacerse una mamografía. Creemos que este cambio resulta de gran interés ya que el médico juega un papel fundamental en el acceso de la mujer a los programas preventivos de cáncer ginecológico ${ }^{16,22}$.

Nuestro estudio muestra que los factores relacionados con actitudes de la mujer e información sobre el cáncer de mama y la mamografía han mejorado sensiblemente en el año 2000 en comparación a 1994. Un mayor número de mujeres muestra actitudes positivas y un mayor nivel de información sobre el cáncer de mama y la mamografía, factores considerados fundamentales para que la mujer acuda a realizarse una mamografía $^{23}$. Para algunos autores la motivación de la mujer por su salud y el conocimiento de la mamografía junto con el riesgo de la enfermedad son los predictores más comunes del uso de la prueba ${ }^{18}$. Llama la atención en nuestro estudio la mayor percepción de riesgo de cáncer de mama en el año 2000 en relación con 1994. Es posible que la mayor difusión de información sobre el cáncer de mama y la mamografía acaecida en los últimos años ${ }^{10,24}$ haya hecho que algunas mujeres puedan sentirse más vulnerables ante la enfermedad.

En cuanto al perfil de las mujeres que se habían realizado una mamografía en los dos años previos no encontramos diferencias significativas en las características sociodemográficas en 1994 y en el año 2000, con excepción de la edad de la mujer. Nuestro estudio muestra un importante incremento en la realización de mamografía en el grupo de 56 a 60 años de edad. Se observa que en el año 2000 la prueba se realiza en mayor medida en mujeres entre 51 y 65 años de edad. Este hecho parece estar en estrecha relación con la existencia de programas de detección precoz de cáncer de mama en las distintas CCAA que suelen incluir como población diana a las mujeres de 50 a 65 años de edad ${ }^{25}$. Observamos que la realización de mamografía es baja en el grupo de más de 65 años de edad, con apenas diferencia entre 1994 y el año 2000. Algunos autores consideran que es preciso prestar atención a estas mujeres ya que la mamografía puede beneficiarlas ${ }^{26}$, y además en Estados Unidos la mitad de los nuevos casos de cáncer de mama son diagnosticados en mujeres por encima de 65 años de edad ${ }^{27}$. La realización de mamografía en el grupo de 40 a 50 años disminuye sensiblemente en el año 2000 en comparación con 1994. Este dato podría interpretarse como una aproximación a las recomendaciones de la Unión Europea que aconsejan la realización de la mamografía en las mujeres mayores de 50 años de edad $^{7}$. No obstante, un tercio de las mamografías realizadas en el año 2000 lo fueron en mujeres de 40 a 50 años, lo que implica una alta proporción de realización de la prueba en un grupo de edad donde los beneficios de la mamografía son más controvertidos ${ }^{28,29}$.

En nuestro estudio no aparecen diferencias de interés en las características sociosanitarias estudiadas de las mujeres con una mamografía en 1994 y el año 2000, con excepción de la recomendación de la mamografía por parte del médico. Este factor aumenta significativamente en el año 2000 con relación a 1994, aunque no en la misma medida que en la población de mujeres de 40 a 70 años de edad. La ausencia de diferencias relevantes en las características sociodemográficas y socio-sanitarias parece indicar un perfil homogéneo de las mujeres que se realizan la prueba.

Al igual que ocurría en la población de mujeres de 40 a 70 años, en el grupo de mujeres con al menos una mamografía en los últimos dos años encontramos en mayor medida actitudes positivas y un mayor nivel 
de información sobre el cáncer de mama y la mamografía en el año 2000 en comparación con 1994. Creemos que el cambio en la actitud de la mujer podría estar en relación con el esfuerzo realizado por las autoridades sanitarias, tanto de la administración autonómica como central, en el desarrollo de actividades encaminadas a la prevención y promoción de la salud de la mujer ${ }^{30,31}$.

A raíz de los datos obtenidos en nuestro estudio consideramos de interés valorar la posible relación entre las variables de actitudes y la edad de la mujer. Comprobamos que la intención de la mujer de hacerse una mamografía en el futuro y la consideración de que la mamografía es necesaria aún sintiéndose bien es mayor en las mujeres jóvenes y en los grupos de edad incluidos en los programas de cribado, y menor en las mujeres mayores de 65 años. Creemos que el aumento de cobertura de los programas hasta los 69 años de edad, tal como están llevando a cabo algunas CCAA, podría ayudar a promover actitudes positivas en las mujeres de esta edad.

Por otra parte, en las mujeres que se habían realizado una mamografía en los dos años previos encontramos una menor proporción de historia de enfermedad de mama en el año 2000 en relación con 1994. Este dato podría indicar la mayor asociación del uso de la mamografía con enfermedades de la mama en 1994, frente a la mayor relación con prácticas preventivas en el año 2000, lo que en parte podría ser debido a los programas de cribado.

En conclusión, el presente estudio muestra que el perfil de las mujeres de 40 a 70 años ha cambiado en España en el año 2000 en relación con 1994. Sin embargo, no ha cambiado sustancialmente el perfil sociodemográfico y sociosanitario de las mujeres que se habían realizado una mamografía en los dos años previos. No obstante, en el año 2000 la realización de la prueba tiene lugar en mayor medida en las mujeres de 51 a 65 años de edad, lo que parece indicar la influencia de los programas de cribado. Resulta sumamente positivo que las mujeres españolas muestren en mayor medida en el año 2000 actitudes positivas y un mayor nivel de información sobre el cáncer de mama y la mamografía.

\section{BIBLIOGRAFÍA}

1. Ferlay J, Bray F, Sankila R, Parkin DM. EUCAN: Cancer Incidence, Mortality and Prevalence in the European Union 1998, version 5.0. IARC CancerBase $N^{\circ}$ 4. Lyon: IARC Press; 1999. [Citado: 1104-05]. Disponible en: http://www-dep.iarc.fr/ eucan/eucan.htm.

2. Centro Nacional de Epidemiología (CNE), Área de Epidemiología Ambiental y Cáncer. Mortalidad por cáncer y otras causas en España, año 2002. Madrid: CNE - Instituto de Salud Carlos III. [Citado: 11-04-05]. Disponible en: http://193.146.50.130/ cancer/mort2002.txt.

3. Banta HD. Health policy, health technology assessment, and screening in Europe. Int J Technol Assess Health Care 2001;17:409-17.

4. Woolf SH. The accuracy and effectiveness of routine population screening with mammography, prostate-specific antigen, and prenatal ultrasound: a review of published scientific evidence. Int J Technol Assess Health Care 2001;17:275-304.

5. Nystrom L, Andersson I, Bjurstam N, Frisell J, Nordenskjold B, Rutqvist LE. Long-term effects of mammography screening: updated overview of the Swedish randomised trials. Lancet 2002; 359:90919.

6. Alonso-Gordo JM. Cáncer de mama. Manejo desde Atención Primaria. Semergen 2000; 26:491-501.

7. Advisory Committee on Cancer Prevention. Recommendations on cancer screening in the European Union. Eur J Cancer 2000; 36:1473-8.

8. Agencia de Evaluación de Tecnologías Sanitarias (AETS). Uso de la mamografía y de la citología de Papanicolau para la detección precoz del cáncer de mama y de cérvix uterino en España. Madrid: AETS - Instituto de Salud Carlos III; 2002. Informe de Evaluación de Tecnologías Sanitarias No 34.

9. Cerdá T, Blanco MC, Corujo M, Queiro T. Programas de cáncer de mama en España. Evolución 
1998-2003. Jornadas de Ámbito Ibérico sobre Programas de Detección Precoz de Cáncer de Mama; Lisboa 2004. [Citado: 11-04-05]. Disponible en: http://ppc.cesga.es/files/lisboa2004/Mesa_Programas/Programas_Espana/Graficos_CCAA_2003.p pt.

10. Instituto Nacional de la Salud, Dirección General de Atención Primaria y Especializada. Plan Integral de Atención a la Mujer. Madrid: INSALUD, Área de Estudios Documentación y Coordinación Normativa; 1998.

11. Luengo S, Lázaro P, Madero R, Alvira F, Fitch K, Azcona B et al. Equity in the access to mammography in Spain. Soc Sci Med 1996; 43:1263-71.

12. Alcaraz M, Lluch A, Miranda J, Pereiro I, Salas MD. Estudio de la no participación en el programa de prevención de cáncer de mama en la ciudad de Valencia. Gac Sanit 2002; 16:230-5.

13. Phillips KA, Kerlikowskw K, Baker LC, Chang SW, Brown ML. Factors associated with women's adherence to mammography screening guidelines. HSR: Health Services Research 1998;33:28-53

14. O’Malley MS, Earp JA, Hawley ST, Schell MJ, Mathews HF, Mitchell J. The association of race/ethnicity, socioeconomic status, and physician recommendation for mammography: Who gets the message about breast cancer screening? Am J Public Health 2001; 91:49-54.

15. Gordon NP, Rundall TG, Parker L. Type of health care coverage and the likelihood of being screened for cancer. Med Care 1998; 36:636-45.

16. Hawley ST, Earp JA, O’Malley M, Ricketts TC. The role of physician recommendation in women's mammography use. Med Care 2000; 38:392-403.

17. Michielutte R, Dignan MB, Smith BL. Psychosocial factors associated with the use of breast cancer screening by women age 60 years or over. Health Educ Behav 1999; 26:625-47.

18. Rutledge DN, Barsevick A, Knobf MT, Bookbinder M. Breast cancer detection: Knowledge, attitudes, and behaviours of women from Pennsylvania. Oncol Nurs Forum 2001; 28:1032-40.

19. Andreu Vaillo Y, Galdón Garrido MJ, Durá Ferrandis E, Carretero Gómez S, Tuells Hernández J. Edad, creencias de salud y asistencia a un programa de cribado mamográfico en la comunidad valenciana. Rev Esp Salud Pública 2004; 78:65-82.
20. Ministerio de Trabajo y Asuntos Sociales. Las mujeres en cifras 2003. Madrid: Instituto de la Mujer. Ministerio de Trabajo y Asuntos Sociales; 2003.

21. Valle Sánchez V. El desarrollo de la España de las autonomías: una perspectiva económica. ICE 2003; 811:133-51. [Citado: 08-04-05]. Disponible en: http://www.upct.es/ de/profesorado/josemiguel/webEcSR/others/ICE8110111.pdf.

22. Borrás JM. Breast cancer screening in Catalonia: cost-effectiveness, health care impact and cost of the treatment of breast cancer. Barcelona: Catalan Agency for Health Technology Assessment; 1996.

23. Meystre-Agustoni G, Dubois-Arber F, De Landstheer J, Paccaud F. Exploring the reasons for non participating of women in a breast cancer screening campaign. Eur J Public Health 1998; 8:143-5.

24. Agencia de Evaluación de Tecnologías Sanitarias (AETS). Cribado poblacional de cáncer de mama mediante mamografía. Rev Calidad Asistencial 1996; 11:184-6.

25. Programas de detección precoz de Cáncer de Mama. [Citado 11-04-05]. Disponible en: http:// ppc.cesga.es

26. Woolf SH. The accuracy and effectiveness of routine population screening with mammography, prostate-specific antigen, and prenatal ultrasound: a review of published scientific evidence. Int J Technol Assess Health Care 2001; 17:275-304.

27. Fox SA, Stein JA, Sockloskie RJ, Ory MG. Targeted mailed materials and the Medicare beneficiary: increasing mammogram screening among the elderly. Am J Public Health 2001; 91:55-61.

28. Moss S. Should women under 50 be screened for breast cancer? Br J Cancer 2004; 91:413-7.

29. Coebergh JW. Challenges and pitfalls of mass-screening in the European Union. Eur J Cancer 2000; $36: 1469-72$

30. Ministerio de Sanidad y Consumo. Criterios mínimos en prevención y promoción de la salud materno-infantil. Madrid: Ministerio de Sanidad y Consumo; 1992.

31. Catálogo de programas en el área de la salud de la mujer y del niño desarrollados por las Comunidades Autónomas. Madrid: Ministerio de Sanidad y Consumo; 1999. 